\title{
CHEBYSHEV POLYNOMIALS AND THE MODULARY GROUP OF LEVEL $p$
}

\author{
R. A. RANKIN
}

1. The inhomogeneous modulary group $\bar{G}(n)$ is the quotient group $\bar{\Gamma}(1) / \bar{\Gamma}(n)$, where $\bar{\Gamma}(1)$ is the full inhomogeneous modular group, and $\bar{\Gamma}(n)$ is the inhomogeneous principal congruence group of level $n$, where $n$ is a positive integer. Each element of $\bar{G}(n)$ can be represented by an infinity of matrices

$$
S=\left(\begin{array}{ll}
a & b \\
c & d
\end{array}\right), \quad a d-b c \equiv 1(\bmod n)
$$

where $a, b, c$ and $d$ are integers, and matrix multiplication is the group operation. If $S$ and

$$
S^{\prime}=\left(\begin{array}{ll}
a^{\prime} & b^{\prime} \\
c^{\prime} & d^{\prime}
\end{array}\right)
$$

represent two elements of the group $\bar{G}(n)$, these elements are not regarded as distinct if and only if

or

$$
a-a^{\prime} \equiv b-b^{\prime} \equiv c-c^{\prime} \equiv d-d^{\prime} \equiv 0(\bmod n),
$$

$$
a+a^{\prime} \equiv b+b^{\prime} \equiv c+c^{\prime} \equiv d+d^{\prime} \equiv 0(\bmod n) \text {, }
$$

which we write symbolically as

$$
S \equiv S^{\prime}(\bmod n) \quad \text { or } \quad S \equiv-S^{\prime}(\bmod n),
$$

respectively. The unit element $\Theta$ of $\bar{G}(n)$ is represented by all matrices $S$ for which

$$
S \equiv \pm I(\bmod n)
$$

where

$$
I=\left(\begin{array}{ll}
1 & 0 \\
0 & 1
\end{array}\right)
$$

In this paper I only consider the groups $\bar{G}(p)$, where $p$ is an odd prime, and write

$$
q=\frac{1}{2}(p-1), \quad r=\frac{1}{2}(p+1) .
$$

Received June 11, 1954. 
It is known that $\bar{G}(p)$ is of order $\frac{1}{2} p\left(p^{2}-1\right)$ and that the order of an element of $\bar{G}(p)$ other than $\Theta$ is either $p$, or a divisor of $q$ or $r$. Further, the elements whose orders divide $p, q$ and $r$ can be divided into $p+1$, $\frac{1}{2} p(p+1)$ and $\frac{1}{2} p(p-1)$ conjugate cyclic subgroups of orders $p, q$ and $r$, respectively, no two of these subgroups having any common elements other than $\Theta$. The $p+1$ subgroups of order $p$ are conjugates of the subgroup generated by

$$
\left(\begin{array}{ll}
1 & 1 \\
0 & 1
\end{array}\right)
$$

and the $\frac{1}{2} p(p+1)$ subgroups of order $q$ are conjugates of the subgroup generated by

$$
\left(\begin{array}{ll}
g & 0 \\
0 & g^{-1}
\end{array}\right)
$$

where $g$ is a primitive root modulo $p$. However the subgroups of order $r$ are not represented so easily, and the only treatments known to me make use of representations in terms of matrices with elements belonging to the Galois field of order $p^{2}$ ([1], [2, pp. 419-491], [3], [4, pp. 363-383 (§§ 464-473)], [5], [6, §§ 101-120]).

It is the purpose of this paper to show how this can be avoided. At the same time the method reveals interesting congruence properties of the Chebyshev polynomials and of the numbers $x$ for which $x^{2}-1$ is either a quadratic residue or non-residue modulo $p$.

2. For any positive integer $n$, and any $\theta$ real or complex, the functions $\cosh n \theta$ and $\sinh n \theta / \sinh \theta$ can be expanded as polynomials of degree $n$ and $n-1$, respectively, in $x=\cosh \theta$; these polynomials we denote by $T_{n}(x)$ and $F_{n}(x)$, respectively. Further, we write $T_{0}(x)=1, F_{0}(x)=0$, and define $T_{-n}(x)=T_{n}(x), F_{-n}(x)=-F_{n}(x)$. The functions $T_{n}$ and $F_{n}$ are recurring sequences in the sense of Lucas and Lehmer. It is easily verified that the following relations hold for all $n$ and $x$. We omit the argument $x$ when no confusion can arise.

$$
\begin{gathered}
F_{n+1}=x F_{n}+T_{n}, \quad F_{n-1}=x F_{n}-T_{n}, \\
F_{n+1}-2 x F_{n}+F_{n-1}=T_{n+1}-2 x T_{n}+T_{n-1}=0, \\
F_{n+1}{ }^{2}-2 x F_{n+1} F_{n}+F_{n}^{2}=1, \\
F_{m n}(x)=F_{m}\left\{T_{n}(x)\right\} F_{n}(x), \quad T_{m n}(x)=T_{m}\left\{T_{n}(x)\right\} \\
T_{2 n-1}-x=2\left(x^{2}-1\right) F_{n-1} F_{n}, \quad T_{2 n-1}+x=2 T_{n-1} T_{n}, \\
T_{n}{ }^{2}-T_{n}^{2}=\left(x^{2}-1\right) F_{m-n} F_{m+n}, \\
T_{n}^{2}=1+\left(x^{2}-1\right) F_{n}^{2}, \quad T_{n}(-1)=(-1)^{n}, \quad T_{n}(1)=1 .
\end{gathered}
$$


Further, $T_{n}(x)$ and $F_{n+1}(x)$ are even functions or odd functions of $x$ according as $n$ is even or odd. For odd $n$ the following expansions hold:

$$
\begin{gathered}
T_{n}(x)= \\
2^{n-1} x^{n}+n \sum_{\nu=1}^{\frac{1}{2}(n-1)}(-1)^{v} \frac{(n-v-1)(n-v-2) \ldots(n-2 v+1)}{v !} 2^{n-2 v-1} x^{n-2 v}, \\
F_{n}(x)=n\left\{1+\sum_{v=1}^{\frac{1}{2}(n-1)} \frac{\left(n^{2}-1^{2}\right)\left(n^{2}-3^{2}\right) \ldots\left\{n^{2}-(2 v-1)^{2}\right\}}{(2 v+1) !}\left(x^{2}-1\right)^{\nu}\right\} .
\end{gathered}
$$

In the second expansion the last term is $2^{n-1}\left(x^{2}-1\right)^{\frac{1}{2}(n-1)}$.

Suppose now that $S$ is defined by (1) where $a, b, c$ and $d$ are integers such that $a d-b c \equiv 1(\bmod p)$, and $p$ is an odd prime. Then it is easily verified by induction, and with the help of (4), that for every positive integer $n$

where

$$
S^{n} \equiv\left(\begin{array}{cc}
a F_{n}-F_{n-1} & b F_{n} \\
c F_{n} & d F_{n}-F_{n-1}
\end{array}\right) \quad(\bmod p)
$$

and $x$ is the argument of the polynomials $F_{n}$ and $F_{n-1}$.

3. In the rest of the paper all congruences are modulo an odd prime $p$. We shall make use of rational numbers in congruences, as is legitimate when the denominators are prime to $p$.

In this section $x$ denotes any integer or residue (not necessarily quadratic) modulo $p$. We use the letter $t$ to stand for either $q$ or $r$, and $s$ to stand for either $p, q$ or $r$. We deduce at once from (10) and (11) that

$$
T_{p}(x) \equiv 2^{p-1} x^{p} \equiv x, \quad F_{p}(x) \equiv 2^{p-1}\left(x^{2}-1\right)^{\frac{1}{2}(p-1)} \equiv\left(x^{2}-1\right)^{\frac{1}{2}(p-1)},
$$

so that we have, by (7) with $p=2 n-1$, for all $x$

$$
\left(x^{2}-1\right) F_{q}(x) F_{r}(x) \equiv 0,
$$

the left-hand member being a polynomial of degree $p$. It follows that the congruences

$$
F_{q}(x) \equiv 0 \quad \text { and } \quad F_{r}(x) \equiv 0
$$

have exactly $q-1$ and $r-1$ solutions, respectively, and have no common solutions. Also, from (3) and (14),

$$
F_{p-1}(x) \equiv x\left\{\left(x^{2}-1\right)^{\frac{1}{2}(p-1)}-1\right\}, \quad F_{p+1}(x) \equiv x\left\{\left(x^{2}-1\right)^{\frac{1}{2}(p+1)}+1\right\} .
$$


Now since, by (6), (7) and (14), $F_{p-1}=2 F_{q} T_{q}, F_{p+1}=2 F_{r} T_{r}$ and $T_{q} T_{r} \equiv 2 x$ it follows that the solutions of $F_{t}(x) \equiv 0$ are, with the possible omission of $x \equiv 0$, the same as those of $F_{2 l}(x) \equiv 0$. Further, since $F_{2}(0)=0$ and $T_{2}(0)=-1$, we have, by $(6)$,

$$
F_{2 m}(0)=F_{m}(-1) F_{2}(0) \equiv 0,
$$

and therefore $x \equiv 0$ is a solution of $F_{t}(x) \equiv 0$ if and only if $t$ is even. For $t=q$ this occurs if and only if $(-1)^{q} \equiv 1$ and, for $t=r$ if and only if $(-1)^{q} \equiv-1$ so that we have, by (14) and (15), the

Theorem 1. For each integer $x$ one and only one of the three congruences hold:

$$
F_{p}(x) \equiv 0, \quad F_{q}(x) \equiv 0, \quad F_{r}(x) \equiv 0 .
$$

In fact the first congruence holds if and only if $x \equiv \pm 1$, the second if and only if $x^{2}-1$ is a quadratic residue modulo $p$, and the third if and only if $x^{2}-1$ is a quadratic non-residue modulo $p$. In particular, $F_{q}(x) \equiv 0$ for $q-1$ incongruent values of $x$ and $F_{r}(x) \equiv 0$ for $r-1$ incongruent values of $x$.

Denote by $\mathscr{C}_{p}, \mathscr{C}_{q}$ and $\mathscr{C}_{r}$ the classes of residues $x$ modulo $p$ for which $F_{p}(x) \equiv 0, F_{q}(x) \equiv 0$ and $F_{r}(x) \equiv 0$, respectively. Note that $\mathscr{C}_{t}$ is empty if and only if $t=q=1$, which occurs for $p=3$. Also, if $x \in \mathscr{C}_{s}$ then $-x \in \mathscr{C}_{s}$.

We define the Chebyshev order $n_{x}$ of a residue $x$ modulo $p$ to be the least positive integer $n$ such that $F_{n}(x) \equiv 0$. We deduce that

$$
T_{n_{x}}(x) \equiv \pm 1: \quad \text { and } \quad T_{n_{x}}(x) \equiv-1 \quad \text { for even } n_{x} .
$$

The first result follows at once from (9), while if for even $n_{x}=2 m$, $T_{2 m}(x) \equiv 1$, we should have

$$
2 T_{m}^{2}=1+T_{2 m} \equiv 2,
$$

and since $F_{2 m}=2 F_{m} T_{m}$, this implies that $\dot{F}_{m}(x) \equiv 0$, which is false.

Now, from (8) with $n=1, T_{m}( \pm 1)= \pm 1$, and, by (6), we therefore have, when $F_{n}(x) \equiv 0$ that

$$
\begin{gathered}
F_{m n+l}=F_{m n} T_{l}+T_{m n} F_{l}=F_{m}\left\{T_{n}(x)\right\} F_{n}(x) T_{l}(x)+T_{m}\left\{T_{n}(x)\right\} F_{l}(x) \\
\equiv T_{m}( \pm 1) F_{t}(x) \equiv \pm F_{l}(x) .
\end{gathered}
$$

We deduce that, if $x \in \mathscr{C}_{s}$, then $n_{x}$ divides $s$, and also that

$$
F_{m}(x) \equiv 0 \quad \text { if and only if } \quad n_{x} \mid m \text {. }
$$


Note that $n_{0}=2$, and that $n_{x}>1$ for all $x$, so that

$$
n_{x}=p \quad \text { for } \quad x \in \mathscr{C}_{p} .
$$

Let $\alpha(n)$ denote the number of incongruent values of $x$ of Chebyshev order $n$ modulo $p$. If for any $t>1$ there exists a residue $x$ modulo $p$ such that the $t-\mathrm{l}$ residues $x=T_{1}(x), T_{2}(x), \ldots, T_{t-1}(x)$ run through all the $t-1$ residues of $\mathscr{C}_{t}$, then we call $x$ a generator of $\mathscr{C}_{t}$.

We prove the following theorem in which $\varphi(n)$ denotes Euler's function.

Theorem 2. (i) We have $\alpha(1)=0, \alpha(p)=2$, and if $n$ is greater than unity and a divisor of $q$ or $r$, then $\alpha(n)=\varphi(n)$. Otherwise $\alpha(n)=0$.

(ii) Each class ' $G_{t}(t>1)$ possesses generators $x$. The number of generators of $\mathscr{C}_{t}(t>1)$ is $\frac{1}{2} \varphi(2 t)$.

(iii) $A$ residue $x$ modulo $p$ is a generator of $C^{C}(t>1)$ if and only if it is of Chebyshev order $t$ and $T_{t}(x) \equiv-1$.

(iv) If $x$ is a generator of $\mathcal{C}_{t}(t>1)$ then the Chebyshev order of $T_{k}(x)$ is $t /(k, t)$, and the full set of generators of $C_{t}$ consists of those residues $T_{k}(x)$ for which $(k, t)=1$ and $k$ is odd.

Proof. To prove (i) it is enough to show that if $n>1$ and if $n$ divides $q$ or $r$ then $\alpha(n)=\varphi(n)$. In the first place, we note that $(q, r)=1$, so that the residues $x$ of Chebyshev order $n$ belong either to $\mathscr{C}_{q}$ or $\mathscr{C}_{r}$, and not to both. Consider therefore the residues $x$ of $\mathscr{C}_{t}(t>1)$ and let $n>1$ be a divisor of $t$. In the first place, the polynomial congruence $F_{n}(x) \equiv 0$, which is of degree $n-1$, has exactly $n-1$ solutions modulo $p$. For let its number of solutions be $k$, where therefore $k \leqq n-1$. Then we have, by (6), putting $t=m n$, that

$$
F_{t}(x)=F_{m}\left\{T_{n}(x)\right\} F_{n}(x) .
$$

Now $F_{m}\left\{T_{n}(x)\right\}$ is a polynomial of degree $(m-1) n=t-n$ in $x$, and so has at most $t-n$ solutions modulo $p$, while $F_{t}(x) \equiv 0$ has exactly $t-1$ solutions. Thus $t-1 \leqq t-n+k$, showing that $k \geqq n-1$. Hence $k=n-1$.

Secondly, if $x$ is of Chebyshev order $d$ where $d$ divides $n$, then $F_{d}(x)$ is a factor of $F_{n}(x)$, so that $F_{n}(x) \equiv 0$. These results show that for every divisor $n$ of $t$ we have

$$
n-1=\sum_{d \mid n} \alpha(d) .
$$

From this, since $\alpha(1)=0$, we deduce, for example by the Möbius conversion formula, that $\alpha(n)=\varphi(n)(n>1)$.

Since $\varphi(t) \geqq 1$, it follows that in each class $C_{t}(t>1)$ there are residues $x$ of order $t$, and that there are, in fact, $q(t)$ of them. Further, if $x$ is of order $t$, so clearly is $-x$. If $t$ is even, then by $(16), T_{t}(x) \equiv-1$ for 
each of these $\varphi(t)$ residues. If, however, $t$ is odd, then $0 \notin^{\prime} \mathscr{C}_{t}$ and, since $T_{t}(x)$ is an odd function of $x$ it follows that, for half of the $\varphi(t)$ residues, $T_{t}(x) \equiv-1$, and for the other half $T_{t}(x) \equiv 1$. Since $\frac{1}{2} \varphi(2 t)$ is $\varphi(t)$ or $\frac{1}{2} \varphi(t)$ according as $t$ is even or odd, part (ii) will follow if we can prove (iii).

We show first that $n_{x}=t$ when $x$ is a generator of $\mathscr{C}_{t}$. For if $n_{x}=n<t$ then, since $n$ divides $t, 2 n-1<t$, and we have, by (7), $T_{2 n-1}(x) \equiv x$, which cannot occur for a generator $x$. Secondly, if $x$ is a generator of $\mathscr{C}_{t}$ then $T_{t}(x) \equiv-1$. For

$$
T_{t-n} \pm T_{n}=T_{n}\left(T_{t} \pm 1\right)-\left(x^{2}-1\right) F_{t} F_{n} \equiv T_{n}\left(T_{t} \pm 1\right),
$$

so that, if, for odd $t>1, T_{t}(x) \equiv 1$ then $T_{t-n}(x) \equiv T_{n}(x)$, and hence, for $1 \leqq n<t$, the residues $T_{n}(x)$ run through at most $\frac{1}{2}(t-1)$ members of $\mathscr{C}_{t}$; thus such a value of $x$ cannot be a generator.

We now suppose that $n_{x}=t$, and that $T_{t}(x) \equiv-1$, and show that $x$ is a generator of $\mathscr{C}_{t}$. For if $1 \leqq n<t$ we have, by (6),

$$
F_{t}\left\{T_{n}(x)\right\} F_{n}(x)=F_{t n}(x)=F_{n}\left\{T_{t}(x)\right\} F_{t}(x) \equiv 0,
$$

so that $F_{t}\left\{T_{n}(x)\right\} \equiv 0$, that is $T_{n}(x) \in \mathscr{C}_{t}$. Hence it remains to show that if $1 \leqq m \leqq n \leqq t$ and $T_{m}(x) \equiv T_{n}(x)$ then $m=n$. By (8), we have

$$
\left(x^{2}-1\right) F_{m-n}(x) F_{m+n}(x) \equiv 0,
$$

so that, by (17), either $t$ divides $m-n$, that is $m=n$, or else $t$ divides $m+n$, that is $m=t-n$. In the latter case we have, by (18),

$$
0 \equiv T_{t-n}-T_{n} \equiv T_{n}\left(T_{t}-1\right) \equiv-2 T_{n} .
$$

Hence $T_{n}(x) \equiv 0$, and therefore

$$
F_{2 n}(x) \equiv F_{2}\left\{T_{n}(x)\right\} F_{n}(x) \equiv F_{2}(0) F_{n}(x) \equiv 0,
$$

which implies that $t=2 n$, that is $m=n$. This completes the proof of (iii).

To prove (iv) suppose that $1 \leqq k<t$ and that $h=(k, t), t=h u$, $k=h v$. Then

$$
0 \equiv F_{t v}(x) \equiv F_{u k}(x) \equiv F_{u}\left\{T_{k}(x)\right\} F_{k}(x),
$$

and therefore $F_{u}\left\{T_{k}(x)\right\} \equiv 0$. Thus $T_{k}(x)$ is of order $n$, say, where $n$ divides $u$.

Conversely, since $F_{n k}(x)=F_{n}\left\{T_{k}(x)\right\} F_{k}(x), n$ is the smallest positive integer such that $t$ divides $n k$, i.e. such that $u$ divides $n v$, i.e. such that $u$ divides $n$, since $(u, v)=1$. Thus $n=u=t /(k, t)$. To complete the proof of (iv) we observe that the residues of order $t$ are $T_{k}(x)$ for $(k, t)=1$, 
and that $T_{k}(x)$ is a generator if and only if, in addition, $k$ is odd. For by (9).

$$
T_{t}\left\{T_{k}(x)\right\}=T_{t k}(x)=T_{k}\left\{T_{t}(x)\right\}=T_{k}(-1)=(-1)^{k},
$$

The following theorem shows, in particular, that if $x$ is a generator of $\mathscr{C}_{t}(t>1)$, then $T_{n}(x)$ and $F_{n}(x)$ are periodic functions of $n$ modulo $p$ with period $2 t$.

Theorem 3. If $x$ is a generator of $\mathscr{C}_{t}(t>1)$, then

$$
\begin{array}{rlrl}
T_{2 t_{ \pm n}}(x) \equiv T_{n}(x), & F_{2 t_{ \pm n}}(x) & \equiv \pm F_{n}(x), \\
T_{t_{ \pm n}}(x) \equiv-T_{n}(x), & F_{t_{ \pm n}}(x) \equiv \pm F_{n}(x) .
\end{array}
$$

We omit the proof, which is straightforward.

We note also that, if $t$ is odd and $x$ is of Chebyshev order $t$, but not a generator of $\mathscr{C}_{t}$, then

$$
\pm T_{1}(x), \pm T_{2}(x), \ldots, \pm T_{\frac{1}{2}(t-1)}(x)
$$

run through all the members of $\mathscr{C}_{t}$ and so provide an alternative method of generating the set.

As an example, consider $p=29$ so that $q=14, r=15$. We find that 5 is a generator of $\mathscr{C}_{14}$ and -2 is a generator of $\mathscr{C}_{15}$. The members of the two classes are given in the following table:

$\begin{array}{lrrrrrrrrrrrrrr}n & 1 & 2 & 3 & 4 & 5 & 6 & 7 & 8 & 9 & 10 & 11 & 12 & 13 & 14 \\ T_{n}(5) & 5 & -9 & -8 & -13 & -6 & 11 & 0 & -11 & 6 & 13 & 8 & 9 & -5 & \\ T_{n}(-2) & -2 & 7 & 3 & 10 & -14 & -12 & 4 & -4 & 12 & 14 & -10 & -3 & -7 & 2\end{array}$

The class $\mathscr{C}_{14}$ has 6 generators, namely $\pm 5, \pm 6, \pm 8(\bmod 29)$, while ${ }^{\prime}{ }_{15}$ has 4 generators, namely $-2,4,-10$ and $-7(\bmod 29)$.

In conclusion, we prove

Theorem 4. If $x$ is a generator of $C_{q}$ and $y$ is chosen so that $x^{2}-1 \equiv y^{2}$, then $x+y$ is a primitive root modulo $p$. Conversely, if $g$ is a primitive root modulo $p$ and $g g^{\prime} \equiv 1$, then $x \equiv \frac{1}{2}\left(g+g^{\prime}\right)$ is a generator of ' $G_{q}$.

Proof. If $x$ is a generator of $\mathscr{C}_{q}$ and $x^{2}-1 \equiv g^{2}$, it is easily shown, by using (3), that

$$
(x+y)^{n} \equiv T_{n}(x)+y F_{n}(x) .
$$

Now $x+y \neq 0$, so that it possesses an order, $k$ say, modulo $p$. Since $(x+y)^{q} \equiv-1, k$ cannot be odd, as otherwise it would divide $q$ and we should obtain a contradiction. Hence $k=2 n$, say, and therefore, by (19), 


$$
0 \equiv(x+y)^{n}-(x+y)^{-n} \equiv\left(T_{n}+y F_{n}\right)-\left(T_{n}-y F_{n}\right) \equiv 2 y F_{n},
$$

from which we deduce that $n=q$, that is, $x+y$ is a primitive root.

Conversely, if $g$ is a primitive root modulo $p$ and $x \equiv \frac{1}{2}\left(g+g^{\prime}\right)$ we can take $y \equiv \frac{1}{2}\left(g-g^{\prime}\right)$ as a solution of $x^{2}-1 \equiv y^{2}$. Then the preceding argument may be reversed to show that $n=q$ is the least positive integer for which $F_{n}(x) \equiv 0$ and that $T_{n}(x) \equiv-1$. Thus $x$ is a generator of $\mathscr{C}_{q}$.

4. Theorem 5. If $\xi$ is an element of the modulary group $\bar{G}(p)(p>2)$ other than the unit element $\Theta$, and if $S$ is any matrix representing $\xi$, then the order of $\xi$ is the same as the Chebyshev order of $x$ modulo $p$ where $x$ is any integer such that $2 x \equiv \operatorname{tr} S$.

Proof. Let the representing matrix $S$ be defined by (1), where $a d-b c \equiv 1$. Then the order $N(\xi)$ of $\xi$ is the least positive integer $N$ such that $S^{N} \equiv \pm I$, and is the same for every matrix representing $\xi$. Thus, by (12), $N$ is the least positive integer for which

$$
a F_{N}-F_{N-1} \equiv d F_{N}-F_{N-1} \equiv \pm 1, \quad b F_{N} \equiv c F_{N} \equiv 0 .
$$

Thus either $N=n_{x}$, since then $F_{N-1} \equiv \pm 1$, by (5), or else $0<N<n_{x}$ and $b \equiv c \equiv 0$. In the second case we deduce that $a \equiv d$ and $a d \equiv 1$, that is $a \equiv d \equiv \pm 1$, which is false since $\xi \neq \Theta$. This proves Theorem 5 .

With the help of Theorem 5 and the results of $\S 3$ we can show that the order of an element of $\bar{G}(p)$ other than $\Theta$ is either $p$ or a divisior of $q$ or $r$, and that the elements whose orders divide $p, q$ or $r$ can be divided into $p+1, \frac{1}{2} p(p+1)$ and $\frac{1}{2} p(p-1)$ conjugate cyclic subgroups of orders $p, q$ and $r$ respectively. Further the $p+1$ subgroups of order $p$ are conjugates of the subgroup generated by

$$
P=\left(\begin{array}{ll}
1 & 1 \\
0 & 1
\end{array}\right)
$$

while the $p(p-t)$ subgroups of order $t(t=\dot{q}$ or $r)$ are conjugates of the subgroup generated by

$$
X=\left(\begin{array}{cc}
x & x+1 \\
x-1 & x
\end{array}\right)
$$

where $x$ is a generator of $\mathscr{C}_{t}$. In proving these results we could at various points refer the reader to [1], [2, pp. 419-491] or [4, pp. 363-383 (§§ 464473)], but prefer to give the argument in full. Also we shall adopt a wholly arithmetic approach and shall not derive the results by considering permutable matrices. 
From Theorem 5 it follows that the elements of $\bar{G}(p)$ can be divided into four classes, (i) the unit element $\Theta$, (ii) $\Im_{p}$, (iii) $\Im_{q}$ and (iv) $\mathfrak{\subseteq}_{r}$, where $\xi \in \mathbb{S}_{s}$ if and only if $\xi \neq \Theta, N(\xi) \mid s$. We denote by $N_{s}$ the number of elements in $\mathfrak{\subseteq}_{s}$.

The class $\mathfrak{S}_{p}$. For every element of $\subseteq_{p}$ we must have $x \in \mathscr{C}_{p}$, where $2 x=\operatorname{tr} S$, that is $x \equiv \pm 1$. Then $2 N_{p}$ is the number of solutions $a, b, c, d$ of the congruences

$$
a+d \equiv \pm 2, \quad b c \equiv a d-1,
$$

the solutions $a \equiv d \equiv \pm 1, b \equiv c \equiv 0$ being excluded. Thus $N_{p}+1$ is the number of solutions of

$$
a+d \equiv 2, \quad b c \equiv a d-1 .
$$

By considering the cases $a d \equiv 1, a d \equiv 1$ separately we easily see that

so that

$$
N_{p}+1=(p-1)(p-1)+2 p-1=p^{2},
$$

$$
N_{p}=p^{2}-1 \text {. }
$$

Since two cyclic subgroups of order $p$ are either identical or have only $\Theta$ in common, and since $p^{2}-1=(p-1)(p+1)$, it follows that the elements of $\Im_{p}$ belong to $p+1$ different cyclic subgroups of $\bar{G}(p)$ of order $p$.

One of these subgroups is that generated by $P$ (see (20)). Let $S$ be any other matrix belonging to $\Im_{p}$ and suppose, as we may, that $a+d=2$. Also, write

$$
T=\left(\begin{array}{ll}
\alpha & \beta \\
\gamma & \delta
\end{array}\right), \quad \alpha \delta-\beta \gamma \equiv \equiv 1,
$$

so that

$$
T^{-1} P^{\lambda} T=\left(\begin{array}{cc}
1+\lambda \gamma \delta & \lambda \delta^{2} \\
-\lambda \gamma^{2} & 1-\lambda \gamma \delta
\end{array}\right)
$$

Suppose first that $c \neq 0$. Then $T^{-1} P^{\lambda} T \equiv S$ if we take

$$
\lambda \equiv \equiv-c, \quad \alpha \equiv 0, \quad \beta \equiv-1, \quad \gamma \equiv 1, \quad \delta \equiv-(a-d) /(2 c) .
$$

If $c \equiv 0$, then $a d \equiv 1$, that is, $a \equiv d \equiv 1$ and $S \equiv P^{b}$. Hence, in either case, $S$ is the transform of a power of $P$ and it follows that the $p+1$ subgroups of order $p$ are conjugate.

The classes $\mathfrak{S}_{q}$, $\mathfrak{S}_{r}$. We can to a large extent treat these together. For every element of $\Im_{t}$ we must have $x \in \mathscr{C}_{t}$. Let $M_{t}(x)$ be the number of solutions of the congruence

$$
a+d \equiv 2 x, \quad b c \equiv a d-1 .
$$


We can have $b c \equiv 0$ only when $a d \equiv 1$, i.e. when $x \equiv a+a^{-1}$. We then have

$$
x^{2}-1 \equiv\left\{\frac{1}{2}\left(a-a^{-1}\right)\right\}^{2},
$$

which, since $a \neq \pm 1$, implies that $x \in \mathscr{C}_{q}$.

Thus, if $x \in \mathscr{C}_{q}$, of the $p$ possible pairs $a, d$ two give $a d \equiv 1$ and so

$$
M_{\boldsymbol{q}}(x)=(p-2)(p-1)+2(2 p-1)=p^{2}+p=2 p(p-t) .
$$

On the other hand, if $x \in \mathscr{C}_{r}$, we cannot have $a d \equiv 1$, and so

Hence

$$
M_{r}(x)=p(p-1)=2 p(p-t) .
$$

$$
N_{t}=\frac{1}{2}(t-1) M_{t}(x)=p(p-t)(t-1) \text {. }
$$

As a check we note that, by (22) and (24),

$$
\begin{aligned}
1+ & N_{p}+N_{q}+N_{r} \\
& =1+\left(p^{2}-1\right)+\frac{1}{4}(p-3) p(p+1)+\frac{1}{4}(p-1) p(p-1)=\frac{1}{2} p\left(p^{2}-1\right),
\end{aligned}
$$

which is the order of $\bar{G}(p)$.

Now suppose that $x$ is a generator of $\mathscr{C}_{t}$, and consider the $2 p(p-t)$ members of $\Im_{t}$ whose representing matrices $S$ have $a+d \equiv 2 x$. Since $\operatorname{tr} S=\operatorname{tr} S^{-1}$, we may group these matrices into $p(p-t)$ pairs $S, S^{\mathbf{- 1}}$. Each pair generates a cyclic group of order $t$ represented by

$$
I, S, S^{2}, \ldots, S^{t-1} \text {. }
$$

No two of these cyclic groups have a common element other than $\Theta$. For if this were not so then we should have

$$
S_{1}{ }^{m} \equiv \pm S_{2}{ }^{n}
$$

for some matrices $S_{1}, S_{2}$ each of trace $2 x$, with

$$
S_{1} \neq S_{2}, \quad S_{1} \neq S_{2}^{-1},
$$

and $1 \leqq m<t, 1 \leqq n<t$. Taking the trace of each side we have

$$
T_{m}(x) \equiv \pm T_{n}(x),
$$

from which it follows, since $x$ is a generator, and by (8) and (18), that either $m=n$ (for + sign), or $m=t-n$ (for - sign). By taking $S_{2}{ }^{-1}$ in place of $S_{2}$, if necessary, we may therefore assume that $m=n$ and that $S_{1}{ }^{n} \equiv S_{2}{ }^{n}$. Hence we have, with an obvious notation,

$$
\begin{gathered}
a_{1} F_{n}-F_{n-1} \equiv a_{2} F_{n}-F_{n-1}, \quad b_{1} F_{n} \equiv b_{2} F_{n}, \\
c_{1} F_{n} \equiv c_{2} F_{n}, \quad d_{1} F_{n}-F_{n-1} \equiv d_{2} F_{n}-F_{n-1} .
\end{gathered}
$$


Since $F_{n} \neq 0$ we deduce that $S_{1} \equiv S_{2}$, which shows that (25) cannot hold.

Further, since it is easily seen that each member of $\mathfrak{S}_{t}$ with trace $2 T_{n}(x) \quad(1 \leqq n<t)$ is the $n$th power of some element with trace $2 x$, we conclude that the elements of $\Im_{t}$ can be divided into $N_{t} /(t-1)=$ $p(p-t)$ cyclic subgroups of order $t$, no two of which possess common elements other than $\Theta$.

Now suppose that $S$ is any matrix (1) with $a d-b c \equiv 1$ and trace $2 x$. We wish to choose a matrix $T$ (see (23), (21)) to make

$$
T^{-1} X T \equiv S \text {. }
$$

This implies that

$$
\begin{gathered}
\gamma \delta(x+1)-\alpha \beta(x-1) \equiv a-x \equiv x-d, \\
\delta^{2}(x+1)-\beta^{2}(x-1) \equiv b, \\
-\gamma^{2}(x+1)+\alpha^{2}(x-1) \equiv c .
\end{gathered}
$$

Suppose first that $c \neq 0$. For different values of $\gamma$ and $\alpha$, each of $\alpha^{2}(x-1)$ and $\gamma^{2}(x+1)+c$ take $\frac{1}{2}(p+1)$ different values modulo $p$, and hence for at least one pair $\alpha, \gamma$, the two expressions are congruent modulo $p$, so that (29) is satisfied. With these values of $\alpha, \gamma$ we can solve (27) together with $\alpha \delta-\beta \gamma \equiv 1$ for $\beta$ and $\delta$, since the determinant of the two congruences is $c \neq 0$. These values must automatically satisfy (28) since $a d-b c \equiv 1$ and $c \neq 0$.

If $c \equiv 0$ and $b \neq 0$ we can similarly find a matrix $T$ by solving first for $\delta$ and $\beta$ and then choosing $\alpha$ and $\gamma$.

Finally, if $b \equiv c \equiv 0$, then $a d \equiv 1$, and so if we take $y \equiv \frac{1}{2}(a-d) \neq 0$, we have $x^{2}-1 \equiv y^{2}$; that is, $x \in \mathscr{C}_{q}$. We now choose

$$
\alpha \equiv y, \quad \beta \equiv \lambda y, \quad \gamma \equiv x-1, \quad \delta \equiv-\lambda(x-1), \quad 2 \lambda y(x-1) \equiv-1
$$

and the congruences (27), (28) and (29) are then all satisfied.

Thus in all cases we can find a $T$ to satisfy (26) for each matrix $S$ of $\varsigma_{t}$, and this shows that the $p(p-t)$ subgroups of order $t$ are selfconjugate.

5. Unsolved problem. For large primes $p$, how small can $|x|$ be, where $x$ is a generator of $\mathscr{C}_{q}$ or $\mathscr{C}_{r}$ ?

\section{REFEREN CES}

1. J. Gierster, Die Untergruppe der Galoisschen Gruppe der Modulargleichung für den Fall eines primzahligen Transformationsgrades, Math. Ann. 18 (1881), 319-365. 
2. F. Klein und R. Fricke, Vorlesungen über die Theorie der elliptischen Modulfunktionen, Band I, Leipzig, 1890.

3. J.-A. Serret, Sur les fonctions rationelles linéaires prise suivant un module premier, et sur les substitutions auxquelles conduit la considération de ces fonctions, C. R. Acad. Sci. Paris 48 (1859), 112-117, 178-182, 237-240.

4. J.-A. Serret, Cours d'algèbre supérieure, tome II, Paris, 1879.

5. G. Vivanti, Elementi della teoria delle funzioni poliedriche e modulari, Milano, 1906.

6. G. Vivanti, Les fonctions polyédriques et modulaires (traduit par A. Cahen), Paris, 1910.

THE UNIVERSITY, BIRMINGHAM, ENGLAND 